\title{
Geometric Interpretation of the Minkowski Metric
}

\author{
Thomas Merz* \\ (Dated: September 4, 2019)
}

\begin{abstract}
A novel geometric interpretation of the Minkowski metric is provided, which offers a different and more intuitive approach to phenomena in special relativity. First it is shown that a change of basis in Minkowski space is the equivalent of a change of basis in Euclidean space if a basis element is replaced by its dual element, constituting a mixed basis set. The methodology of the proof includes infinitesimal changes of basis using the Lie-algebras of the involved groups. As a consequence, a direct mapping between Euclidean and Minkowski space is defined. Second, a measuring device called a local, flat observer is defined in Euclidean space and it is shown, that this device uses a mixed basis when measuring distances. Combining these steps, it is concluded that a local, flat observer in a four-dimensional Euclidean spacetime measures a Minkowski spacetime.
\end{abstract}

\section{INTRODUCTION}

Special relativity can be formulated within the fourdimensional Minkowski space $\mathbb{R}(3,1)$, which is defined by the following scalar product:

$$
\begin{gathered}
\mathbf{d v} \cdot \mathbf{d} \mathbf{w}=\sum_{\mu \nu} g_{\mu \nu} d v^{\mu} d w^{\nu} \\
\text { with } \quad g_{\mu \nu}=[S 1] \cdot \operatorname{diag}(-1,+1,+1,+1) .
\end{gathered}
$$

The covariant tensor $g_{\mu \nu}$ is called Minkowski metric, $\mathbf{d v}$ and $\mathbf{d w}$ can be any four-vector elements of the Minkowski space. The algebraic sign $[S 1]= \pm 1$ can be assigned freely depending on the convention [1.

Although this description is elegant and successful, it does not allow a direct geometrical interpretation of the underlying quantities. A direct geometric/ trigonometric interpretation in the Euclidean sense is not possible because the scalar product defined by $g_{\mu \nu}$ is not positive definite, which in turn implies that the norm induced by the scalar product $\|\mathbf{d v}\|=\sqrt{\mathbf{d v} \cdot \mathbf{d v}}$ can assume imaginary values (Figure 1). This means that imaginary distances between two points can occur in the vector space, and there is no intuitive trigonometric equivalent for this.

This is seen as a significant shortcoming for authors who seek geometric interpretations of the underlying systems of equations (e.g. 22 and references included therein; [3], [4], [5], 6]).

The purpose of this article is to offer this missing piece needed to acknowledge geometrical theories of spacetime. In Section II it is shown that the Minkwowski metric, rather than simply being a direct property of spacetime, could appear when a four-dimensional Euclidean space is measured with a mixed basis $\left\{\mathbf{e}^{0}, \mathbf{e}_{1}^{*}, \mathbf{e}_{2}^{*}, \mathbf{e}_{3}^{*}\right\}$.

The question arising from this finding is what kind of instrumental setup or observer would use such a mixed measuring procedure. To answer it, Section III considers the hypothesis of a flat observer who has no spatial extent in one dimension, but who may still be able to

\footnotetext{
*t.merz@alumni.ethz.ch http://elasticuniverse.org/
}

measure this additional dimension indirectly by using a contravariantly transforming scale.

\section{MAPPING BETWEEN SPACES WITH MIXED COVARIANT AND CONTRAVARIANT BASES}

\section{A. Basics and notation}

In the following, co- and contravariant vectors are written in bold, second order tensors are written in bold and have capital letters; greek indexes run from 0 to 3, latin indexes from 1 to 3 , unless otherwise noted. To avoid confusion, elements of the dual space and their components are marked with a ()*

Let $\mathbb{R}^{4}$ be a four-dimensional Euclidean vector space with states $\mathbf{v} \in \mathbb{R}^{4}$. These states can be expressed as a linear combination of a canonical basis $\left\{\mathbf{e}_{\mu}^{*}\right\} \in \mathbb{R}^{4 *}$ with elements from the dual space (orig. [7, available at [8]; introductions e.g. in [9, [10]):

$$
\begin{aligned}
\mathbf{v} & =\sum_{\mu} \mathbf{e}_{\mu}^{*} x^{\mu} \quad \text { where } \\
x^{\mu} & =\left(\mathbf{v}^{*} \cdot \mathbf{e}^{\boldsymbol{\mu}}\right)=\sum_{\nu}\left(v_{\nu}^{*} \cdot\left(e^{\mu}\right)^{\nu}\right) \quad \mathbf{x}=\left(\begin{array}{l}
x^{0} \\
x^{1} \\
x^{2} \\
x^{3}
\end{array}\right) .
\end{aligned}
$$

The scalar coefficients $x^{\mu}$ are called coordinates of the state $\mathbf{v}$ with respect to the basis $\left\{\mathbf{e}_{\boldsymbol{\mu}}^{*}\right\}$. They can be summarized as a coordinate vector $\mathbf{x}$ (Eq. 2 last term). The coordinates are generated by the dual basis $\left\{\mathbf{e}^{\mu}\right\} \in$ $\mathbb{R}^{4}$, which is defined by $\left(e_{\mu}^{*}\right)_{\nu}=\sum_{\xi} g_{\nu \xi}^{E u k l .}\left(e^{\mu}\right)^{\xi}$ with the Euclidean metric tensor $g_{\nu \xi}^{E u k l .}=\delta_{\nu \xi}$.

Analogously, any dual state $\mathbf{w}^{*} \in \mathbb{R}^{4 *}$ can be generated 


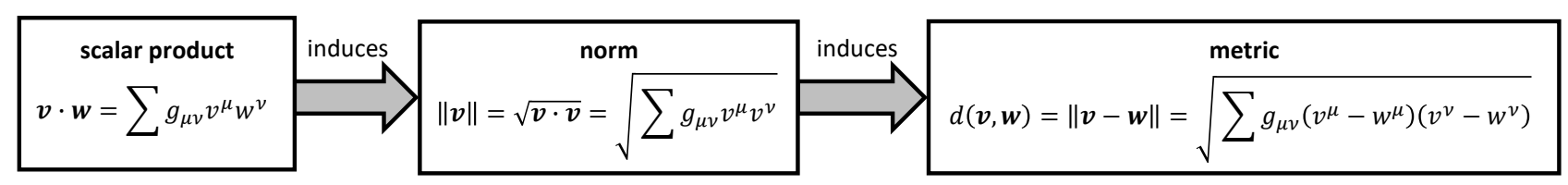

FIG. 1. Relation between scalar product, induced norm and metric.

by a canonical dual basis:

$$
\begin{aligned}
\mathbf{w}^{*} & =\sum_{\mu} y_{\mu}^{*} \mathbf{e}^{\mu} \quad \text { where } \\
y_{\mu}^{*} & =\left(\mathbf{e}_{\mu}^{*} \cdot \mathbf{w}\right)=\sum_{\nu}\left(\left(e_{\mu}^{*}\right)_{\nu} \cdot w^{\nu}\right) \\
\mathbf{y}^{*} & =\left(\begin{array}{llll}
y_{0}^{*} & y_{1}^{*} & y_{2}^{*} & y_{3}^{*}
\end{array}\right) .
\end{aligned}
$$

The coefficients $y_{\mu}^{*}$ are called dual coordinates of the dual state $\mathbf{w}$ with respect to the dual basis $\left\{\mathbf{e}^{\mu}\right\}$. They can be consolidated as a dual coordinate vector $\mathbf{y}^{*}$ (Eq. 3 last row).

Thus, scalar and tensor products can be directly defined:

$$
\mathbf{y}^{*} \cdot \mathbf{x}=\sum_{\mu} y_{\mu}^{*} x^{\mu} \in \mathbb{R} \quad \mathbf{x} \otimes \mathbf{y}^{*}=\mathbf{M} \in \mathbb{R}^{4 \times 4}
$$$$
\text { with } \quad M_{\nu}^{\mu}=x^{\mu} y_{\nu}^{*}
$$

The state $\mathbf{v}$ does not vary when there's a change of basis. For this to be the case, the coordinates $\mathbf{x}$ must change inversely to the basis.

Changes of orthonormal bases in the four-dimensional Euclidean space are isomorphic to the Lie-group $S O(4)$, which can be represented by the special orthogonal matrices of fourth order $S O(4)=\left\{\mathbf{R} \in G L(4) \mid \mathbf{R}^{\mathbf{T}} \mathbf{R}=\mathbb{1}\right.$, $\operatorname{det} \mathbf{R}=1\}$. Consider the change of basis from $\left\{\mathbf{e}_{v}^{*}\right\}$ to $\left\{\mathbf{e}_{\mu}^{\prime *}\right\}$. The elements of the new basis can be written as a linear combination of the elements of the old basis:

$$
\mathbf{e}_{\mu}^{* *}=\sum_{\nu} \mathbf{e}_{v}^{*} R_{\mu}^{\nu}
$$

This translates into the following transformation rule for the coordinate vectors:

$$
\begin{aligned}
\mathbf{x}^{\prime} & =\mathbf{R}^{-1} \cdot \mathbf{x}=\mathbf{R}^{\mathbf{T}} \cdot \mathbf{x} \\
x^{\prime \mu} & =\sum_{\xi}\left(R^{-1}\right)_{\xi}{ }^{\mu} x^{\xi}=\sum_{\xi} R_{\xi}^{\mu} x^{\xi} .
\end{aligned}
$$

The transpose of the matrix $\mathbf{R}$ is expressed by the index interchange. The inverse transformation of the coordinates is true for any change of basis; the fact that this corresponds to the transposed transformation matrix is a consequence of the orthonormality of the considered bases. The components of the transformation matrix and its inverse are:

$$
R_{\mu}{ }^{\nu}=\mathbf{e}_{\mu}^{* *} \cdot \mathbf{e}^{v} \quad\left(R^{-1}\right)_{\mu}{ }^{\nu}=R_{\mu}^{\nu}=\mathbf{e}_{\mu}^{*} \cdot \mathbf{e}^{\prime v}
$$

It is said that the basis $\left\{\mathbf{e}_{\boldsymbol{\mu}}^{*}\right\}$ transforms covariantly, whereas the coordinates $\mathbf{x}$ transform contravariantly. As required, the state $\mathbf{v}$ remains unchanged:

$$
\begin{aligned}
\mathbf{v}^{\prime} & =\sum_{\mu} \mathbf{e}_{\mu}^{*} x^{\prime \mu}=\sum_{\mu, \nu, \xi} \mathbf{e}_{\nu}^{*} R_{\mu}{ }^{\nu} R_{\xi}^{\mu} x^{\xi} \\
& =\sum_{\nu, \xi} \mathbf{e}_{v}^{*} \delta_{\xi}^{\nu} x^{\xi}=\sum_{\nu} \mathbf{e}_{v}^{*} x^{\nu}=\mathbf{v} .
\end{aligned}
$$

\section{B. Definitions}

Definition 1 (mixed basis). A mixed basis $\left\{\mathbf{e}^{\mathbf{0}}, \mathbf{e}_{\mathbf{1}}^{*}, \mathbf{e}_{\mathbf{2}}^{*}, \mathbf{e}_{\mathbf{3}}^{*}\right\}$ is defined as a basis where all elements belong to the canonical basis, except one which is replaced by its dual element, with $\left(e_{0}^{*}\right)_{\nu}=\sum_{\xi} g_{\nu \xi}^{E u k l .}\left(e^{0}\right)^{\xi}$.

Definition 2 (mixed change of basis). Let a mixed change of basis be the change of basis between two mixed bases $\left\{\mathbf{e}^{\mathbf{0}}, \mathbf{e}_{\mathbf{1}}^{*}, \mathbf{e}_{\mathbf{2}}^{*}, \mathbf{e}_{\mathbf{3}}^{*}\right\}$ and $\left\{\mathbf{e}^{\prime 0}, \mathbf{e}_{\mathbf{1}}^{\prime *}, \mathbf{e}_{\mathbf{2}}^{* *}, \mathbf{e}_{\mathbf{3}}^{\prime *}\right\}$.

Remark 1. It is clear that mixed bases as in Definition 1 do not change like regular bases in Euclidean space; nor can the scalar or tensor product of the Euclidean space be applied to the mixed bases.

The question now is whether it is possible to define a vector space in which the mixed bases can be described in a coherent mathematical manner. Hence the following proposition: 


\section{Proposition and proof}

Proposition 1. A mixed basis $\left\{\mathbf{e}^{\mathbf{0}}, \mathbf{e}_{\mathbf{1}}^{*}, \mathbf{e}_{\mathbf{2}}^{*}, \mathbf{e}_{\mathbf{3}}^{*}\right\}$ in Euclidean space as the one in Definition 1 undergoing mixed changes of basis as the ones in Definition 2 behaves just like a regular basis $\left\{\mathbf{f}_{\boldsymbol{\mu}}\right\}$ would in the Minkowski space, where the scalar product is defined as:

$$
\begin{aligned}
\mathbf{y} \cdot \mathbf{x} & =\sum_{\mu \nu} g_{\mu \nu} y^{\mu} x^{\nu} \\
\text { with } \quad g_{\mu \nu} & =\operatorname{diag}(-1,+1,+1,+1) .
\end{aligned}
$$

Proof. Let $\mathbb{R}^{4}$ be the Euclidean space with the canonical basis $\left\{\mathbf{e}_{\boldsymbol{\mu}}^{*}\right\}$ and dual basis $\left\{\mathbf{e}^{\boldsymbol{\mu}}\right\} ; \mathbb{R}(3,1)$ the Minkowski space with the orthonormalized basis $\left\{\mathbf{f}_{\boldsymbol{\mu}}^{*}\right\}$. It has to be shown that, when doing a change of basis, the element $\mathbf{e}^{\mathbf{0}}$ of the dual Euclidean basis transforms like the element $\mathbf{f}_{\mathbf{0}}^{*}$ of the Minkowski basis.

The regular changes of basis in Euclidean space $S O(4)=\left\{\mathbf{R} \in G L(4) \mid \mathbf{R}^{\mathbf{T}} \mathbf{R}=\mathbb{1}, \operatorname{det} \mathbf{R}=1\right\}$ can be expressed as an exponential series:

$$
\mathbf{R}=e^{t \mathbf{A}} \quad \mathbf{R}^{-1}=\mathbf{R}^{\mathbf{T}}=e^{-t \mathbf{A}}
$$

Where $t$ is the parameter of the transformation that could be interpreted as a rotation angle and $\mathbf{A}$ is a skew symmetric matrix, where $\mathbf{A}^{\mathbf{T}}=-\mathbf{A}$. The skew symmetry of these matrices is what finally leads to the orthogonality of the transformations and the coordinate vectors to change with the transposed transformation rule. More precisely, the matrices $\mathbf{A}$ build, together with the commutator $[\mathbf{A}, \mathbf{B}]=\mathbf{A B}-\mathbf{B A}$, the Lie-algebra $s o(4)$ of the Lie-group $S O(4)$ : the algebra that generates all infinitesimal orthogonal linear coordinate transformations, which through the exponential mapping span the whole of the $S O(4)$ Lie-group (for introductions see e.g. [11], [12]).

In the case of infinitesimal transformations ( $t$ very small) it is sufficient to only consider the first terms of the exponential series. The special role of the Lie-algebra is seen here:

$$
\mathbf{R}=e^{t \mathbf{A}} \approx(\mathbf{1}+t \mathbf{A}) \quad \mathbf{R}^{-\mathbf{1}}=e^{-t \mathbf{A}} \approx(\mathbf{1}-t \mathbf{A})
$$

The elements $\mathbf{A}$ of the Lie-algebra can once more be expressed as a linear combination of a basis, which in the case of so(4) consists of six skew symmetrical matrices, e.g.:

$$
\mathbf{L}_{\mathbf{1}}=\left(\begin{array}{cccc}
0 & 0 & 0 & 0 \\
0 & 0 & 0 & 0 \\
0 & 0 & 0 & -1 \\
0 & 0 & 1 & 0
\end{array}\right) \mathbf{L}_{\mathbf{2}}=\left(\begin{array}{cccc}
0 & 0 & 0 & 0 \\
0 & 0 & 0 & 1 \\
0 & 0 & 0 & 0 \\
0 & -1 & 0 & 0
\end{array}\right) \mathbf{L}_{3}=\left(\begin{array}{cccc}
0 & 0 & 0 & 0 \\
0 & 0 & -1 & 0 \\
0 & 1 & 0 & 0 \\
0 & 0 & 0 & 0
\end{array}\right)
$$

$$
\mathbf{K}_{\mathbf{1}}=\left(\begin{array}{cccc}
0 & -1 & 0 & 0 \\
1 & 0 & 0 & 0 \\
0 & 0 & 0 & 0 \\
0 & 0 & 0 & 0
\end{array}\right) \mathbf{K}_{\mathbf{2}}=\left(\begin{array}{cccc}
0 & 0 & -1 & 0 \\
0 & 0 & 0 & 0 \\
1 & 0 & 0 & 0 \\
0 & 0 & 0 & 0
\end{array}\right) \mathbf{K}_{\mathbf{3}}=\left(\begin{array}{cccc}
0 & 0 & 0 & -1 \\
0 & 0 & 0 & 0 \\
0 & 0 & 0 & 0 \\
1 & 0 & 0 & 0
\end{array}\right)
$$

$$
\begin{array}{ll}
\text { with } & {\left[\mathbf{L}_{\mathbf{i}}, \mathbf{L}_{\mathbf{j}}\right]=\varepsilon_{i j k} \mathbf{L}_{\mathbf{k}}} \\
& {\left[\mathbf{K}_{\mathbf{i}}, \mathbf{K}_{\mathbf{j}}\right]=\varepsilon_{i j k} \mathbf{L}_{\mathbf{k}}} \\
& {\left[\mathbf{L}_{\mathbf{i}}, \mathbf{K}_{\mathbf{j}}\right]=\varepsilon_{i j k} \mathbf{K}_{\mathbf{k}}}
\end{array}
$$

and therefore

$$
t \mathbf{A}=\sum_{i=1,2,3}\left(t^{i} \mathbf{L}_{\mathbf{i}}+s^{i} \mathbf{K}_{\mathbf{i}}\right)
$$

To apply a change of the mixed basis, a transformation rule has to be constructed which transforms the element $\mathbf{e}_{\mathbf{0}}$ of the basis with the same coefficients that a coordinate vector $\mathbf{e}^{\mathbf{0}}$ is transformed with. The infinitesimal change of a normalized coordinate vector $\mathbf{e}^{\mathbf{0}}=(1,0,0,0)^{\mathbf{T}}$ can be written as follows:

$$
\begin{aligned}
\mathbf{e}^{\mathbf{0}} & =(\mathbf{1}-t \mathbf{A}) \mathbf{e}^{\mathbf{0}} \\
& =\left(\mathbf{1}-\sum_{i=1,2,3} s^{i} \mathbf{K}_{\mathbf{i}}\right) \mathbf{e}^{\mathbf{0}} \\
& =\left(\mathbf{1}+\sum_{i=1,2,3} s^{i} \mathbf{K}_{\mathbf{i}}^{-\mathbf{1}}\right) \mathbf{e}^{\mathbf{0}}
\end{aligned}
$$

or in componentwise notation:

$$
\begin{aligned}
\left(e^{\prime 0}\right)^{\nu} & =\left(\delta_{0}^{\nu}-t A_{0}^{\nu}\right)\left(e^{0}\right)^{0} \\
& =\left(\delta_{0}^{\nu}-\sum_{i=1,2,3} s^{i}\left(K_{i}\right)_{0}{ }^{\nu}\right)\left(e^{0}\right)^{0} \\
& =\left(\delta_{0}^{\nu}+\sum_{i=1,2,3} s^{i}\left(K_{i}^{-1}\right)_{0}^{\nu}\right)\left(e^{0}\right)^{0} .
\end{aligned}
$$

Where the (pseudo-)inverse $\mathbf{K}_{\mathbf{i}}^{\mathbf{- 1}}$ is the inverse on the subspace spanned by $\mathbf{e}_{\mathbf{i}}^{*}$ and $\mathbf{e}_{\mathbf{0}}^{*}$. 
On the other hand, the transformation rule for $\mathbf{e}_{\mathbf{0}}^{*}$ is:

$$
\begin{aligned}
\mathbf{e}_{\mathbf{0}}^{* *} & =\sum_{\nu} \mathbf{e}_{\boldsymbol{v}}^{*}\left(\delta_{0}{ }^{\nu}+t A_{0}{ }^{\nu}\right) \\
& =\sum_{\nu} \mathbf{e}_{v}^{*}\left(\delta_{0}{ }^{\nu}+\sum_{i=1,2,3} s^{i}\left(K_{i}\right)_{0}{ }^{\nu}\right) .
\end{aligned}
$$

For the new mixed transformation to be useful, it must again function as a Lie algebra. Although it cannot be presumed that such a Lie algebra exists, if a corresponding algebra is found that fulfills the requirements, its existence is proven automatically.

Looking for a Lie algebra, it must satisfy by definition the transformation rule 17. By definition of the base transformations, the coordinates must also satisfy equation 16 .

In order for $\mathbf{e}_{0}^{*}$ to transform additionally in the same way as $\mathbf{e}^{\mathbf{0}}$, according to equations 16 (last term) and 17 (last term), the following must hold true: $\left(K_{i}^{-1}\right)_{0}{ }^{\nu}=$ $\left(K_{i}\right)_{0}{ }^{\nu}$. Each $\mathbf{K}_{\mathbf{i}}$ must therefore be its inverse in the subspace spanned by itself.

This condition cannot be fulfilled by regular Euclidean transformations, since in this case the $\mathbf{K}_{\mathbf{i}}$ are skew symmetric, $\mathbf{K}_{\mathbf{i}}^{-1}=-\mathbf{K}_{\mathbf{i}}$.

To nonetheless find a change of basis which has the same effect on the element of the basis $\mathbf{e}_{0}^{*}$ as it has on the coordinate vector $\mathbf{e}^{\mathbf{0}}$, one must depart from changes of basis in the ordinary Euclidean sense. Consider a new transformation rule (marked with a tilde):

$$
t \widetilde{\mathbf{A}}=\sum_{i=1,2,3}\left(t^{i} \mathbf{L}_{\mathbf{i}}+\tilde{s}^{i} \widetilde{\mathbf{K}}_{\mathbf{i}}\right)
$$

Different elements $\widetilde{\mathbf{K}}_{\mathbf{i}}$ of the basis are sought, which are their own inverses in their own spanned subspace. A possible choice is:

$\widetilde{\mathbf{K}}_{1}=\left(\begin{array}{llll}0 & 1 & 0 & 0 \\ 1 & 0 & 0 & 0 \\ 0 & 0 & 0 & 0 \\ 0 & 0 & 0 & 0\end{array}\right) \widetilde{\mathbf{K}}_{\mathbf{2}}=\left(\begin{array}{llll}0 & 0 & 1 & 0 \\ 0 & 0 & 0 & 0 \\ 1 & 0 & 0 & 0 \\ 0 & 0 & 0 & 0\end{array}\right) \widetilde{\mathbf{K}}_{3}=\left(\begin{array}{llll}0 & 0 & 0 & 1 \\ 0 & 0 & 0 & 0 \\ 0 & 0 & 0 & 0 \\ 1 & 0 & 0 & 0\end{array}\right)$

The new elements retain the form of the transformations for $\mathbf{e}_{\mathbf{0}}^{*}$, yet without orthogonality, since the skew symmetry of the Lie-algebra's elements had to be abandoned.

Hence, a transformation rule was constructed which takes a basis of the Euclidean space $\left\{\mathbf{e}_{\mu}^{*}\right\}$ and changes it in the way a mixed basis $\left\{\mathbf{e}^{\mathbf{0}}, \mathbf{e}_{1}^{*}, \mathbf{e}_{2}^{*}, \mathbf{e}_{3}^{*}\right\}$ would change when exposed to regular Euclidean changes of basis. The contravariant transformation property of $\left\{\mathbf{e}^{\mathbf{0}}\right\}$ was hereby transferred to $\left\{\mathbf{e}_{\mathbf{0}}\right\}$.

The unchanged matrices $\left\{\mathbf{L}_{\mathbf{i}}\right\}$ build, together with the new matrices $\left\{\widetilde{\mathbf{K}}_{\mathbf{i}}\right\}$ and the commutator as Lie bracket, the Lie-algebra $s o(3,1)$, with its elements $\widetilde{\mathbf{A}} \in \operatorname{so}(3,1)$. By means of the exponential mapping this Lie-Algebra translates into the Lorentz group $S O(3,1)$, where:

$$
\boldsymbol{\Lambda}=e^{t \widetilde{\mathbf{A}}} \quad \boldsymbol{\Lambda} \in S O(3,1) .
$$

Yet the elements of the Lorentz group $\boldsymbol{\Lambda}$ are defined as those changes of basis taking place within Minkowski space $\mathbb{R}(3,1)$, having metric tensor $g_{\mu \nu}=\operatorname{diag}(-1,+1,+1,+1)$.

Thus, if one creates a transformation in Euclidean space, where one of the elements of the Euclidean basis transforms contravariantly, the defined basis will be equivalent to a regular basis of the Minkowski space and the discovered transformation will be equivalent to regular changes of bases in the Minkowski space.

\section{INTERPRETATION AS DISTANCE MEASUREMENT BY A FLAT OBSERVER}

\section{A. Definitions}

Definition 3 (Local observer). Let $\mathbb{R}^{4}$ be a fourdimensional Euclidean vector space with the metric tensor $g_{\mu \nu}^{E u k l .}=\operatorname{diag}(+1,+1,+1,+1)$ and the metric

$d(\mathbf{d} \mathbf{v}, \mathbf{d w})=\|\mathbf{d} \mathbf{v}-\mathbf{d} \mathbf{w}\|=\sqrt{\sum_{\mu \nu} g_{\mu \nu}^{E u k l .}\left(d v^{\mu}-d w^{\mu}\right)\left(d v^{\nu}-d w^{\nu}\right)}$.

In this space, a local observer is defined as a device that performs distance measurements within the infinitesimal neighborhood $\varepsilon$ of a point $P$.

Definition 4 (Flat observer). Let $M$ be a be a connected, analytic submanifold of this vector space $\mathbb{R}^{4}$, where $\operatorname{dim}(M)=3$.

A flat observer is defined as a local observer at the point $P_{M}$ according to Definition 3 , with the restriction that the flat observer is an element of $M$, and his measurement neighborhood $\varepsilon_{M}$ is part of $M$, thus $P_{M} \in M$ and $\varepsilon_{M} \in$ M.

Corollary 1. The measurement neighborhood $\varepsilon_{M}$ of a flat observer can thus be defined through the tangential space of the submanifold at the place of the observer $P_{M}$.

Since $M$ is analytical and connected, a canonical basis $\left\{\mathbf{e}_{1}^{*}, \mathbf{e}_{2}^{*}, \mathbf{e}_{3}^{*}\right\}$ can be found for the flat observer, where $\mathbf{e}_{\mathbf{0}}^{*}$ is normal to $M$.

Remark 2. Only local measurements by a single observer are treated in this article.

Definition 5 (Measurable objects). Measurable objects are constructs which trigger a measurable signal that can be evaluated by a local observer according to Definition 3 . 


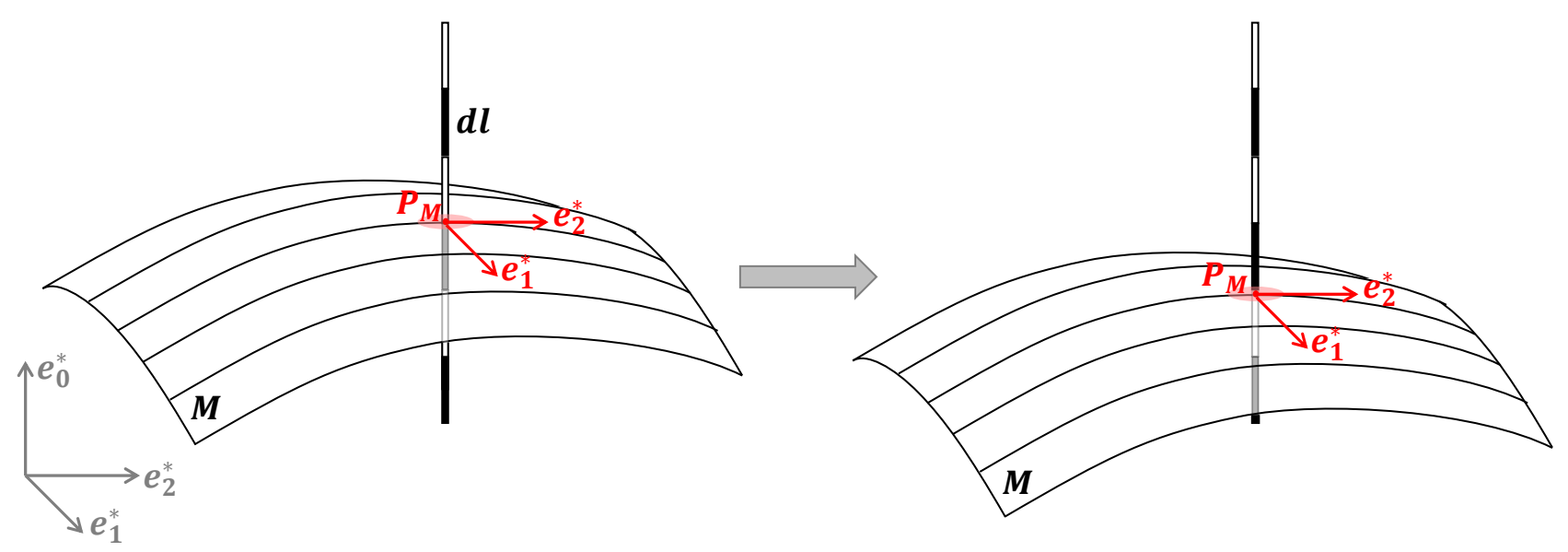

FIG. 2. Illustrative three-dimensional example: an infinitesimally thin rod in the $\mathbf{e}_{\mathbf{0}}^{*}$ direction, consisting of black and white elements of length $d l$. The flat observer at the point $P_{M}$ in a submanifold $M$ with basis $\left\{\mathbf{e}_{1}^{*}, \mathbf{e}_{2}^{*}\right\}$ (red) measures the thin rod only as a point or very small disk. The observer can determine whether the disk is black or white. Now imagine the flat observer and the rod moving relative to each other along the $\mathbf{e}_{\mathbf{0}}^{*}$ direction (situation on the right). By counting the black and white elements that have passed through, the flat observer is given a measuring rule for distances which are not in his submanifold. In this example, the length of a black or white element serves as the standard length, the count corresponds to determining any distance as a multiple of the standard length. On the bottom left in grey the basis system $\left\{\mathbf{e}_{0}^{*}, \mathbf{e}_{1}^{*}, \mathbf{e}_{2}^{*}\right\}$ of a local observer is shown, whose measurement is not limited to a submanifold.

Corollary 2. According to Equation 21, the only quantities which can be inputted in a measurement procedure carried out by a local observer are vectors, thus measurable objects must be inherently defined as vectorial quantities.

\section{B. Proposition and proof}

By definition, a flat observer cannot directly take distance measurements along the direction normal to his submanifold (parallel to $\mathbf{e}_{\mathbf{0}}^{*}$ ).

Under certain conditions, however, indirect distance measurements in this direction may become possible:

Lemma 1. A flat observer according to Definition 4 can make distance measurements in the $\mathbf{e}_{\mathbf{0}}^{*}$ direction if:

A) A measurable object $\mathbf{O}$ with spatial extent in the $\mathbf{e}_{\mathbf{0}}^{*}$ direction is present, and it intersects the measurement neighborhood $\varepsilon_{M}$ of the flat observer $(\mathbf{O} \cap$ $\left.\varepsilon_{M} \neq\{\}\right)$, and

$B)$ there is relative displacement between $\varepsilon_{M}$ and the object in the $\mathbf{e}_{\mathbf{0}}^{*}$ direction.

Proof. A) To perform any measurement the metric in Equation 21 must be used. Measurable objects are defined as input quantities for this metric. They are also the only way the flat observer can measure distances in the $\mathbf{e}_{\mathbf{0}}^{*}$ direction, since a basis vector is not available. Hence, for the measurement in this direction, a measurable object $\mathbf{O}$ must be present.
To measure a distance other than zero in some direction, the two vectors inserted into the metric must differ in the coordinate of interest. A length $d(\mathbf{d v}, \mathbf{d w})$ normal to $M$ can only be defined by two vectors $\mathbf{d v}, \mathbf{d w}$ with different values in the $\mathbf{e}_{\mathbf{0}}$ component. This difference between the two vectors $\mathbf{d v}$ - dw can just as well be defined as a measurable object $\mathbf{O}$ with a non zero spatial extent in the $\mathbf{e}_{\mathbf{0}}^{*}$ direction. Finally, a flat observer can only perform measurements in his neighborhood $\varepsilon_{M}$, thus the measurable object must intersect that neighborhood.

B) Since the flat observer only has the overview of one single coordinate in the $\mathbf{e}_{\mathbf{0}}^{*}$ direction, a relative shift between $\mathbf{O}$ and $\varepsilon_{M}$ is necessary to observe at least two different coordinate values.

For further illustration of this concept, see the threedimensional example in Figure 2 .

Lemma 2. The measurement of a distance normal to the submanifold according to Lemma 1 by a flat observer according to Definition 4 produces a contravariantly transforming scale.

Proof. For a flat observer, the only measurable connection to the outside of its submanifold $M$ is given by the measurable object $\mathbf{O}$. The basis vector $\mathbf{e}_{\mathbf{0}}^{*}$ is by definition not available for measurement.

Since there are no further references, a standard length in the $\mathbf{e}_{\mathbf{0}}^{*}$ direction can only be defined using the object $\mathbf{O}$ and any additional distance measurement can then be 


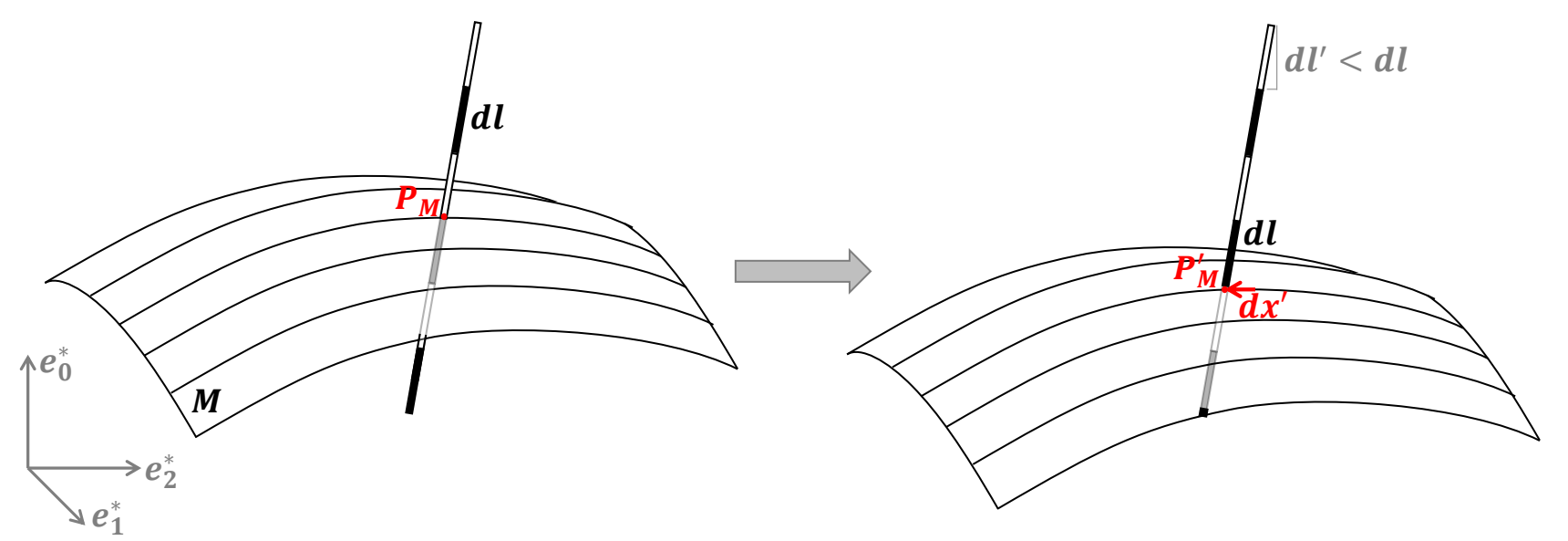

FIG. 3. Illustrative example: an infinitesimally thin rod tilted at a small angle from the $\mathbf{e}_{\mathbf{0}}^{*}$ direction. From the situation on the left to the situation on the right there is a relative displacement between the submanifold of the flat observer $M$ and the rod. After defining the distance measurement outside of its submanifold $M$ by counting black and white elements on the rod, the flat observer has to assume that the distance from black (situation on the left) to white (situation on the right) has remained the same as in Figure 2 In addition the observer has covered the distance $d x^{\prime}$ within his submanifold to follow the rod. Thus, according to this measuring rule, the total distance covered is given by $d s^{\prime 2}=d l^{2}+d x^{\prime 2}$, which can be expected when using a mixed basis and choosing the length element $\mathbf{e}^{\mathbf{0}}=\mathbf{d l}$ as the basis vector for lengths outside of $M$. In contrast, the local observer can use the basis vector $\mathbf{e}_{\mathbf{0}}^{*}$ as a reference and finds that the distance traveled in the $\mathbf{e}_{\mathbf{0}}^{*}$ direction is slightly shorter than the one in Figure $2\left(d l^{\prime}<d l\right)$. The local observer thus makes Euclidean measurements $d s^{\prime 2}=d l^{\prime 2}+d x^{\prime 2}=d l^{2}($ indicated in gray).

quantified as multiples of this standard length. However, the measured standard length used as the basis element and the resulting scale transform contravariantly, since a measurable object $\mathbf{O}$ is a vectorial quantity, as stated in Corollary 2 .

The effect of a contravariant measurement is illustrated in Figure 3 with a three-dimensional example.

It is now possible to formulate the following proposition:

Proposition 2. It is possible to interpret the Minkowski spacetime as a four-dimensional Euclidean space measured by a flat observer according to Definition 4

Proof. The proof is divided into two parts. The first part is to show that the Minkwoski metric can be understood as a measurement rule in an Euclidean space if a mixed basis $\left\{\mathbf{e}^{\mathbf{0}}, \mathbf{e}_{1}^{*}, \mathbf{e}_{2}^{*}, \mathbf{e}_{3}^{*}\right\}$ is used when carrying out the measurement. This part was proven in Section IIC.

The second part is covered by Lemma 2 that shows that a flat observer uses such a mixed basis, where the scale for distance measurement is covariant within his submanifold and contravariant when normal to his submanifold.

Thus, the argumentation is consistent and Proposition 2 is proven.

\section{CONCLUSION}

It has been shown that a flat observer according to Definition 4 uses a mixed basis according to Definition 1 to measure lengths in an additional dimension. This leads to a measurement using a Minkowski metric.

The significance of this result lies in physics: physical experiments measure a Minkowski spacetime. According to the presented result, they behave like flat observers do in a four-dimensional Euclidean spacetime. Therefore, it can be concluded that an Euclidean spacetime may not be just a mathematical trick - as usually suggested when applying similar concepts like e.g. the Wick rotation 13 . - but may be a physical reality, measured by experiments as flat observers.

Consequences can be expected for geometric theories of spacetime (e.g. 2] and references included therein; 3], [4, [5], 6]) as well as for the interpretation of symmetries which have clear geometric interpretations in fourdimensional Euclidean space (e.g. the $S U(2)$ symmetry and related groups, whose interpretation becomes that of a 3 -spherical symmetry).

Geometrically, changing the sign of an element of the metric tensor corresponds to switching the role of basis and coordinate in the affected component. Since in physics vectors and covectors are interpreted differently, this finding may have an effect on choosing the correct sign of the applied metric within a physical theory, since $g_{\mu \nu}=\operatorname{diag}(-1,+1,+1,+1)$ and $\breve{g}_{\mu \nu}=$ $\operatorname{diag}(+1,-1,-1,-1)$ have different interpretations ac- 
cording to the presented results.

On the mathematical side, a transformation which changes mixed bases into each other according to Definition 2 was created. To do so, orthogonality had to be abandoned and Euclidean lengths are no longer preserved. It has been shown that changes between mixed bases are transferable to the Minkowski space, where a coherent description, preserving the Minkowski length element, is possible. This result corresponds to a novel mapping between Euclidean and Minkowski space.

An important realization of this mapping is that all effects arising within the Minkowski space can be explained by purely metric quantities - in other words, quantities obtained through a measurement of distance. The cause of the effects observed can thus be directly transferred to the act of measurement within a mixed basis.

On my private homepage http://elasticuniverse. org an analogous concept with further applications to physics can be found in unreviewed form. The proof developed here, in form of an earlier, less accurate version, is employed.

However, some questions remain unanswered: How can the mapping be applied in real (finite) problems, how can it be used for calculations, and what influence does the topology of the two involved spaces have?

Furthermore the presented mapping is not a homomorphism, it might not be bijective or linear and must be examined in more detail.

\section{ACKNOWLEDGMENTS}

My thanks go to Rudolf Fehlmann for the countless, fruitful discussions and in particular for his support in the structuring of my work. Likewise, my thanks go to Benjamin Arner for the translations into English, as well as to Nelson Bolivar and Ernesto Fuenmayor for the prepublication review. Then to my father for the editing, plus everyone else surrounding me, my family and friends, for the ongoing support.
[1] C. Misner, K. S. Thorne, J. A. Wheeler. Gravitation; W. H. Freeman and Company: San Francisco, US, 1973; ISBN 978-0-691-17779-3.

[2] T. G. Tenev, M. F. Horstemeyer, The Mechanics of Spacetime - A Solid Mechanics Perspective on the Theory of General Relativity. International Journal of Modern Physics D 2018, 27, 1850083. CrossRef

[3] L. Levrino, A. Tartaglia, From the elasticity theory to cosmology and vice versa. A. Sci. China Phys. Mech. Astron. 2014, 57, 597. CrossRef

[4] R. F. Klinkhamer, G. Volovik, Tetrads and q-theory. arXiv:1812.07046 [gr-qc] 2018. CrossRef

[5] H. Kleinert, J. Zaanen, Nematic world crystal model of gravity explaining absence of torsion in spacetime. Physics Letters A 2004, 324, 361-365. CrossRef.

[6] Q. Si-ming, W. You-lin, T4 Gauge Theory of Gravity and New General Relativity. Gen Relat Gravit 1990, 22, 511. CrossRef
[7] J. J. Sylvester, On the general theory of associated algebraical forms. Cambridge and Dublin Math. Journal 1851, VI, 289-293.

[8] J. J. Sylvester. The collected mathematical papers of James Joseph Sylvester; Cambridge University Press: Cambridge, UK, 2012; ISBN 978-1-107-66143-1.

[9] M. Bartelmann, B. Feuerbacher, T. Krüger, D. Lüst, A. Rebhan, A. Wipf. Theoretische Physik; Springer-Verlag, 2014; ISBN 978-3-642-54618-1.

[10] H. G. Evertz, W. von der Linden. Quantenmechanik; TU Graz: Graz, AT, 2017; Link.

[11] B. Hall. Lie Groups, Lie Algebras, and Representations: An Elementary Introduction, Edition 2; Springer-Verlag, 2015; ISBN 978-3-319-13467-3.

[12] K. Walter. Darstellungstheorie der Lorentz-Gruppe; Universität Münster: Münster, DE, 2008; Link.

[13] G. C. Wick, Properties of Bethe-Salpeter Wave Functions. Physical Review 1954, 96 (4): 1124-1134. CrossRef 